\title{
Color and Shape Index for Region-Based Image Retrieval
}

\author{
B.G. Prasad ${ }^{1 \star}$, S.K. Gupta ${ }^{2}$, and K.K. Biswas ${ }^{2}$ \\ 1 Department of Computer Science and Engineering, \\ P.E.S.College of Engineering, Mandya, 571402, INDIA. \\ 2 Department of Computer Science and Engineering, \\ Indian Institute of Technology, New Delhi, 110016, INDIA. \\ \{bgprasad, skg, kkb\}@cse.iitd.ernet.in, \\ WWW home page: http://www.cse.iitd.ernet.in/ ${ }^{\text {skgupta/ }}$
}

\begin{abstract}
Most CBIR systems use low-level visual features for representation and retrieval of images. Generally such methods suffer from the problems of high-dimensionality leading to more computational time and inefficient indexing and retrieval performance. This paper focuses on a low-dimensional color and shape based indexing technique for achieving efficient and effective retrieval performance. We propose a combined index using color and shape features. A new shape similarity measure is proposed which is shown to be more effective. Images are indexed by dominant color regions and similar images form an image cluster stored in a hash structure. Each region within an image is further indexed by a region-based shape index. The shape index is invariant to translation, rotation and scaling. A JAVA based query engine supporting query-byexample is built to retrieve images by color and shape. The retrieval performance is studied and compared with a region-based shape indexing scheme.
\end{abstract}

\section{Introduction}

The past few years have seen many advanced techniques evolving in ContentBased Image Retrieval (CBIR). Applications like medicine, entertainment, education, manufacturing, etc. make use of vast amount of visual data in the form of images. This envisages the need for fast and effective retrieval mechanisms in an efficient manner. A major approach directed towards achieving this goal is the use of low-level visual features of the image data to segment, index and retrieve relevant images from the image database. Recent CBIR systems based on features like color, shape, texture, spatial layout, object motion, etc., are cited in [1], 2]. Of all the visual features, color is the most dominant and distinguishing one in almost all applications.

^ This work is partly supported by the AICTE Young Teachers Career Award 


\subsection{Previous Work in CBIR}

Current CBIR systems such as IBM's QBIC [3], 4] allow automatic retrieval based on simple characteristics and distribution of color, shape and texture. But they do not consider structural and spatial relationships and fail to capture meaningful contents of the image in general. Also the object identification is semi-automatic. The Chabot project [5] integrates a relational database with retrieval by color analysis. Textual meta-data along with color histograms form the main features used. VisualSEEK [6] allows query by color and spatial layout of color regions. Text based tools for annotating images and searching is provided. A new image representation which uses the concept of localized coherent regions in color and texture space is presented by Carson et al. 7]. Segmentation based on the above features called "Blobworld" is used and query is based on these features.

Some of the popular methods to characterize color information in images are color histograms [8],9], color moments [10] and color correlograms [1]. Though all these methods provide good characterization of color, they have the problem of high-dimensionality. This leads to more computational time, inefficient indexing and performance. To overcome these problems, use of SVD [9], dominant color regions approach [12, [13] and color clustering [14] have been proposed.

\subsection{Recent Work in Shape-Based CBIR}

Shape also is an important feature for perceptual object recognition and classification of images. It has been used in CBIR in conjunction with color and other features for indexing and retrieval.

Shape description or representation is an important issue both in object recognition and classification. Many techniques, including chain code, polygonal approximations, curvature, fourier descriptors and moment descriptors have been proposed and used in various applications [15. Recently, techniques using shape measure as an important feature have been used for CBIR. Features such as moment invariants and area of region have been used in [3, 16], but do not give perceptual shape similarity. Cortelazzo [17] used chain codes for trademark image shape description and string matching technique. The chain codes are not normalized and string matching is not invariant to shape scale. Jain and Vailaya [18] proposed a shape representation based on the use of a histogram of edge directions. But these are not scale normalized and computationally expensive in similarity measures. Mehrotra and Gary [19] used coordinates of significant points on the boundary as shape representation. It is not a compact representation and the similarity measure is computationally expensive. Jagadish [20] proposed shape decomposition into a number of rectangles and two pairs of coordinates for each rectangle are used to represent the shape. It is not rotation invariant.

A region-based shape representation and indexing scheme that is translation, rotation and scale invariant is proposed by Lu and Sajjanhar [21]. It conforms to human similarity perception. They have compared it to Fourier descriptor model 
and found their method to be better. But, the images database consists of only 2D planar shapes and they have considered only binary images. Moreover, shapes with similar eccentricity but different shapes are retrieved as matched images. Our aim is to extend this method to represent color image regions and augment the color index of our previous work [13] with the shape features. Our shape indexing feature and similarity measure is different and shown to be effective in retrieval. A combined index based on color and shape has been implemented to improve retrieval efficiency and effectiveness.

The paper is organised as follows: Section 2 describes the color and shape features used for indexing. The indexing scheme, querying and similarity measure are explained in section 3. Section 4 highlights the results of our approach and sample output. A comparision of performance with the scheme employed in [21] is also covered in this section.

\section{Color and Shape Features}

The initial step in our approach is to index images based on dominant color regions [13. Image regions thus obtained after segmentation and indexing are used as input to the shape module. The region-based shape representation proposed in [21] is modified to calculate the shape features required for our proposed shape indexing technique and similarity measure. It is simple to calculate and robust. We show that the retrieval effectiveness is better compared to their method.

\subsection{Color Indexing Approach}

To segment images based on dominant colors, a color quantization in RGB space using 25 perceptual color categories is employed as is done in [13. From the segmented image we find the enclosing minimum bounding rectangle (MBR) of the region, its location, image path, number of regions in the image, etc., and all these are stored in a metafile for further use in the construction of an image index tree. An index tree for the entire database is constructed when the query engine is initiated. At query time, similar images matched on the basis of color and spatial location are retrieved. To the above color index, we have included a region-based shape index similar to the one in 21] which is invariant to rotation, scaling and translation. Since their representation is suited only for 2-D binary image regions, we have used a different shape feature to index the color regions and also a suitable shape similarity measure. A comparision of the two techniques has been carried out for an image database consisting of flags, flowers, fruits, vegetables and simulated shape regions.

\subsection{Shape Representation}

Definitions of terminology:

- Major axis: it is the straight line segment joining the two points on the boundary farthest away from each other (in case of more than one, select any one). 
- Minor axis: it is perpendicular to the major axis and of such length that a rectangle with sides parallel to major and minor axes that just encloses the boundary can be formed using the lengths of the major and minor axes.

- Basic rectangle: the above rectangle formed with major and minor axes as its two sides is called basic rectangle.

- Eccentricity: the ratio of the major to the minor axis is called eccentricity of the region.

- Centroid or Center of gravity: a single point of an object/region towards which other objects/regions are gravitationally attracted. For 2D shapes, the coordinates $\left(X_{c}, Y_{c}\right)$ of the centroid are defined as:

$$
\begin{aligned}
& X_{c}=\sum_{x} \sum_{y} f(x, y) x / \sum_{x} \sum_{y} f(x, y) \\
& Y_{c}=\sum_{x} \sum_{y} f(x, y) y / \sum_{x} \sum_{y} f(x, y)
\end{aligned}
$$

where $(x, y)$ are pixel coordinates and $f(x, y)$ is set to 1 for points within or on the shape and set to 0 elsewhere.

Basic idea. Given a shape region, a grid space consisting of fixed-size square cells is placed over it so as to cover the entire shape region as shown in figure 1 We assign a "1" to cells with at least $25 \%$ of pixels covered and " 0 " to each of the other cells. A binary sequence of 1's and 0's from left to right is obtained as the shape feature representation. For example, the shape in the above figure can be represented by a binary sequence 11111111011111110011011000000110 0000001000000000 .

The smaller the grid size, the more accurate the shape representation is and more the storage and computation requirements. The representation is compact, easy to obtain and translation invariant. Hence, a scale and rotation normalization is carried out to make it invariant to scale and rotation.

Rotation normalization. The purpose of rotation normalization is to place shape regions in a unique common orientation. Hence the shape region is rotated such that its major axis is parallel to the $\mathrm{x}$-axis.

There are still two possibilities as shown in figure 1 and 2, caused by $180^{\circ}$ rotation. Further, two more orientations are possible due to the horizontal and vertical flips of the original region as shown in figures 3 and 4 respectively. Two binary sequences are needed for representing these two orientations. But only

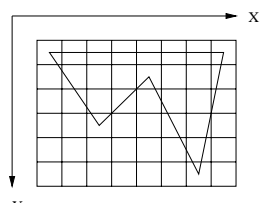

Fig. 1. Grid on region overlayed.

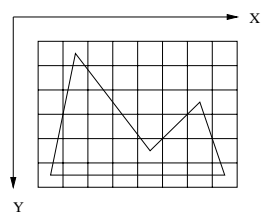

Fig. 2. Region rotated by $180^{\circ}$.

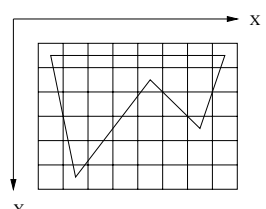

Fig. 3. Horizontal flip of region.

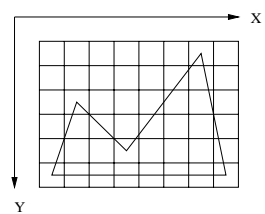

Fig. 4. Vertical flip of region. 
one sequence is stored and at the time of retrieval we can account for these two sequences.

Scale normalization. To achieve scale normalization, we proportionally scale all the shape regions so that their major axes have the same length of 96 pixels.

Shape index. Once the shape region has been normalized for scale and rotation invariance, using a fixed size of grid cells (say 8x8), we obtain a unique sequence for each shape region. The grid size in our proposed method is kept as $96 \mathrm{x}$ 96 pixels. Each sub-grid cell is of size 12x12 pixels giving a binary sequence of length 64 bits per shape region. Using this sequence, we find both the row and column totals of the $8 \times 8$ grid and store them as our shape index, which is more robust and gives a better perceptual representation to the coverage of the shape. A suitable shape similarity measure using this index is employed for matching images at query time.

\section{Indexing Scheme and Querying}

\subsection{Color Index}

A composite index based on a color look-up table is formed consisting of 25 colors. The index is unique and given by the equation below:

$$
\text { Index }=\sum_{i=1}^{N o-R G N} C_{i} * 25^{n-i}
$$

Suppose $(\mathrm{C} 1, \mathrm{C} 2, \mathrm{C} 3)$ are the color indices of three dominant regions found within an image, where $\mathrm{C} 1$ represents index of the first dominant region, $\mathrm{C} 2$ represents index of the second dominant region and $\mathrm{C} 3$ represents index of the third dominant region.

Then, the index is given by

$$
\text { Index }=C 1 * 25^{2}+C 2 * 25^{1}+C 3 * 25^{0}
$$

Images with similar indices are stored in same hash entry of the hash table structure. Each entry also stores the color region features such as location, area, percentage of color, etc., associated to each region of the image which is used in the matching criteria.

\subsection{Shape Index}

For each color region processed above, we compute the shape descriptor as follows:

1. Compute the major and minor axes of each color region. 
2. Rotate the shape region to align the major axis to $\mathrm{X}$-axis to achieve rotation normalization and scale it such that major axis is of standard fixed length (96 pixels).

3. Place the grid of fixed size (96x96 pixels) over the normalized color region and obtain the binary sequence by assigning 1's and 0's accordingly.

4. Using the binary sequence, compute the row and column total vectors. These along with the eccentricity form the shape index for the region.

\subsection{Querying}

Given a query image, we apply the same process on the query image to obtain the color and shape features. Our implementation supports both Query-by-example and Query-by-feature for color matching. The shape matching module supports only Query-by-example. Based on the color index of the query image, a list of matching images are retrieved from the hash structure. Then the shape descriptors are used to find matching images from this initial set to retrieve the final images matched on both color and shape.

The query process is as follows:

1. The query image is processed to obtain a list of matching images based only on color features.

2. For each color region in the query image, the shape representation of each region is evaluated. To take care of the problem of $180^{\circ}$ rotation and vertical and horizontal flips, we need to store 4 sets of the shape index.

3. Compare the shape index of regions in the query image to those in the list of images retrieved on color.

4. Regions with only matching eccentricity within a threshold (t) are compared for shape similarity.

5. The matching images are ordered depending on the difference in the sum of the difference in row and column vectors between query and matching image.

\subsection{Similarity Measure}

Let $R$ and $R^{\prime}$ represent the row vectors of test image and query image respectively. Similarly, $C$ and $C^{\prime}$ represent the column vectors of the test image and query image respectively. The similarity measure is computed as follows:

1. Calculate the row and column vectors of all the regions in the query image.

2. Find the row and column difference between query image regions and regions in the image to be tested using the equation:

$$
\begin{aligned}
& R_{d}=\sum_{i}\left(\left|R_{i}-R_{i}^{\prime}\right|\right) \\
& C_{d}=\sum_{i}\left(\left|C_{i}-C_{i}^{\prime}\right|\right)
\end{aligned}
$$

where $R_{d}$ and $C_{d}$ are the row and column differences between the test image and query image region, $R_{i}$ and $C_{i}$ are the $i^{t h}$ bit of row and column vectors in image and $R_{i}^{\prime}$ and $C_{i}^{\prime}$ are the $i^{\text {th }}$ bit of row and column vectors in the query image.

3. If $\left(R_{d}+C_{d}\right)<T$ (threshold), then the images match. 


\section{Experimental Results and Performance}

The experimental database consists of about 200 images of flags and 120 images of fruits, flowers and simulated objects(squares, rectangles, triangles, circles, etc). Each image in the database is indexed on color and shape features. A hash table stores images of similar index based on the features extracted. Images are retrieved first based on the color index and displayed. Then shapes of all regions in the query image are compared to the region shapes in the displayed set to find images similar on the basis of shape index.

An example output for retrieval for the image database of flowers, fruits and simulated shape regions is shown in figure 5 for matching on color and in figure 6 for matching on shape. It can be observed that images non-similar in shape are eliminated. The image on the left of the screen is the query image. Figures [7 and 8 show the corresponding results for the image database of flags.

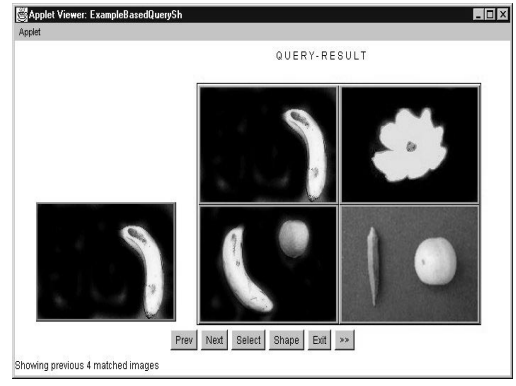

Fig. 5. Retrieval of images based on matching color regions

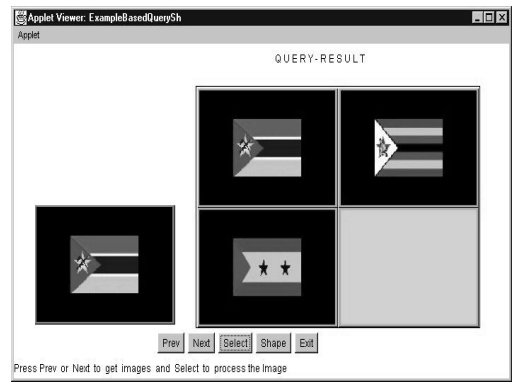

Fig. 7. Retrieval of images based on matching color regions

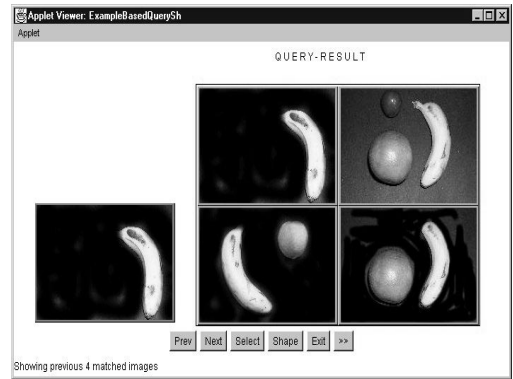

Fig. 6. Retrieval of images based on matching shape regions

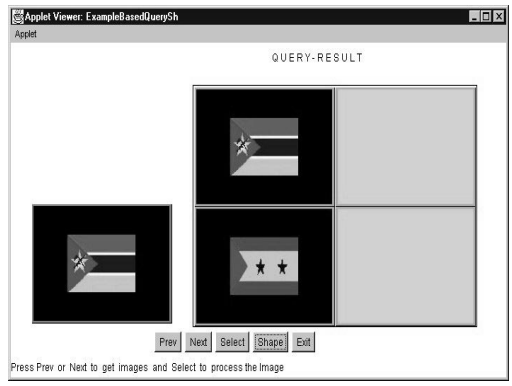

Fig. 8. Retrieval of images based on matching shape regions 


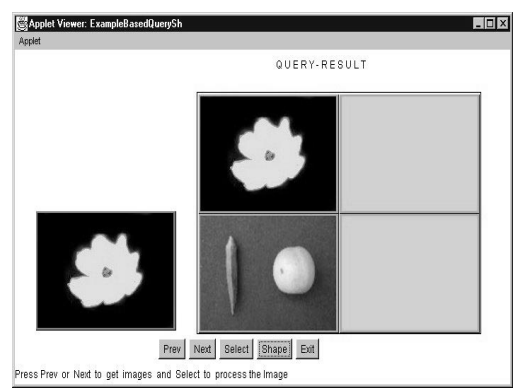

Fig. 9. Retrieval results based on similarity measure of 21 .

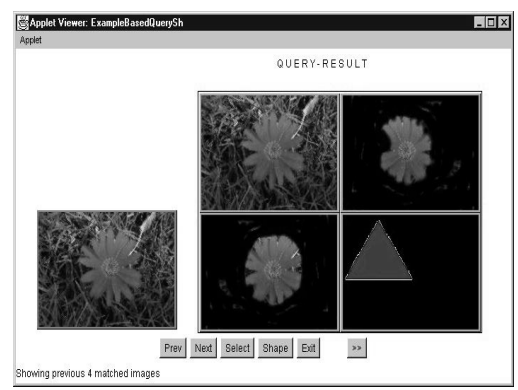

Fig. 11. Retreival results based on similarity measure of [21].

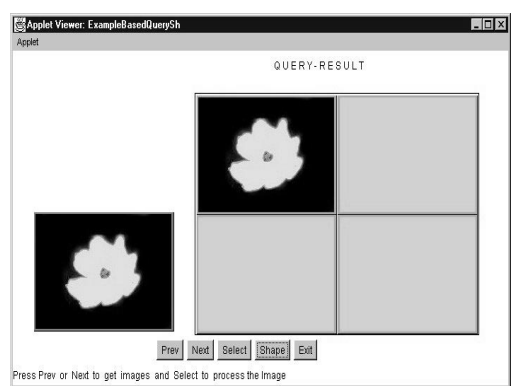

Fig. 10. Retrieval results based on our proposed similarity measure.

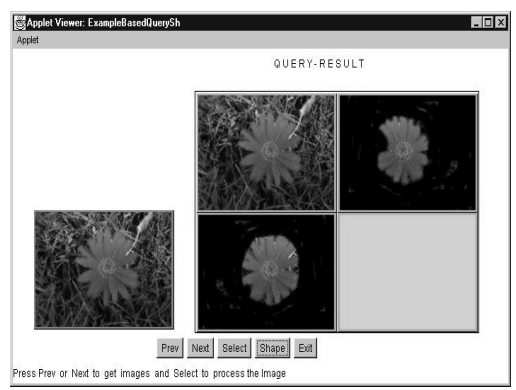

Fig. 12. Retrieval results based on our proposed similarity measure.

We have compared the results of our technique with that proposed in [21]. The output for the two comparative techniques are shown in figures 9 and 10 for the image database of flowers, fruits and simulated shape regions. Figures 11 and 12 show the corresponding difference in retrieval results for the flag image database. The outputs show that there is better pruning of the matched images using the row and column vector based technique for matching images.

The retireval performance is measured using recall and precision, as is standard in all CBIR systems. Recall measures the ability of retrieving all relevant or similar items in the database. It is defined as the ratio between the number of relevant or perceptually similar items retrieved and the total relevant items in the database. Precision measures the retrieval accuracy and is defined as the ratio between the number of relevant or perceptually similar items and the total number of items retrieved.

The graph in figure 13 shows the retrieval performance for the image database of flowers, fruits and simulated shapes in terms of color and shape curves. Similar analysis is done for the flag image database and shown in figure 14

A comparative study of the two different techniques of indexing and retrieval is shon in figures 15] and 16 respectively. It is seen that our indexing method and similarity measure provides better retrieval effectiveness. 


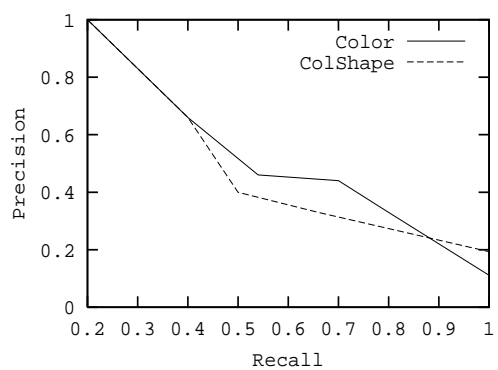

Fig. 13. Recall-precision graph for image database of flowers etc.

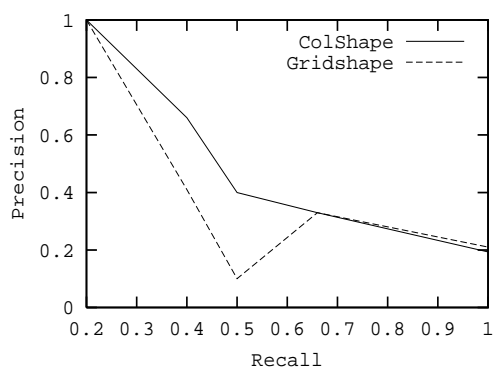

Fig. 15. Recall-Precision comparing the two similarity measures for image database of flowers, etc.

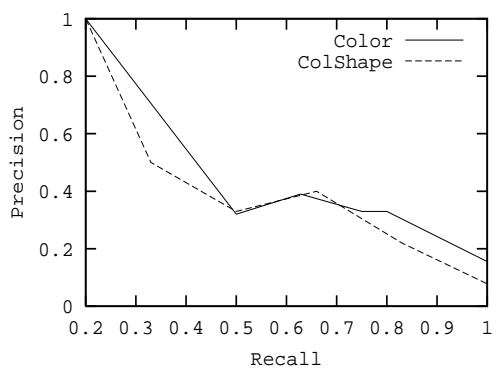

Fig. 14. Recall-precision graph for image database of flags.

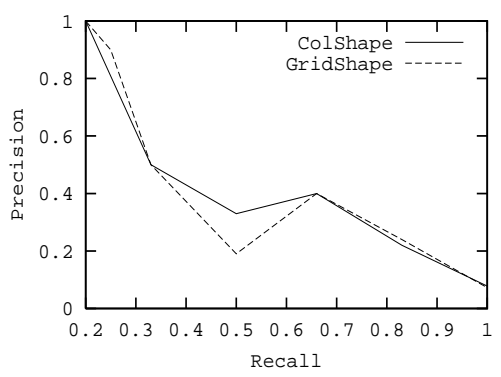

Fig. 16. Recall-Precision comparing the two similarity measures for image database of flags.

\section{Conclusions}

A combined color and shape based low-dimensional indexing technique has been implemented. Images are segmented into dominant regions based on perceptually similar color regions using a color quantized indexing method. Such segmented out regions are stored in a hash structure as similar image clusters. Shape features of these regions are used to further prune the retrieval of images from a sample image database. The shape representation is based on a grid-based coverage of the region which is normalized to achieve invariance in scale, rotation and size. The index is a robust one. Our proposed index based on row and column vectors and the related similarity measure is shown to provide an efficient and effective retrieval performance. A JAVA based search engine using query-by-example has been developed on Windows-NT platform. The results and performance analysis of our method shows that it is effective and efficient. It can be further enhanced by including texture features. 


\section{References}

1. V.N.Gudivada and V.V.Raghavan.: Special issue on content-based image retrieval systems - guest eds. IEEE Computer. 28(9) (1995) 18-22

2. M.De Marsicoi, L.Cinque, and S.Levialdi.: Indexing pictorial documents by their content: a survey of current techniques. Image and Vision Computing. 15(2) (1997) 119-141

3. M.Flickner etal.,: Query by image and video content: the qbic system. IEEE Computer. 28(9) (1995) 23-32

4. W.Niblack etal.,: The qbic project: Querying images by content using color, texture and shape. In Storage and Retrieval for Image and Video Databases (SPIE). 1908 $173-187$

5. V.E.Ogle and M.Stonebaker.: Chabot: Retrieval from a relational database of images. IEEE Computer. 28(9) (1995) 40-48

6. J.R.Smith and S.F.Chang.: Visualseek: A fully automated content-based image query system. ACM Multimedia. (1996) 87-98

7. C.Carson etal.,: Region-based image querying.: In CVPR'97 Workshop on Contentbased Access to Image and Video libraries (CAIVL'97) (1997)

8. M.J.Swain and D.H.Ballard.: Color indexing. Intl. Journal of Computer Vision. Vol. 7 No. 1 (1991) 11-32

9. J.Hafner etal.,: Efficient color histogram indexing for quadratic form distance functions. PAMI. Vol. 17 No. 7 July. (1995) 729-736

10. M.Stricker and A.Dimai.: Color indexing with weak spatial constraints. Proceedings of SPIE Storage and Retrieval of Still Image and Video Databases IV. Vol. 2670 (1996) 29-40

11. J.Huang etal.,: Image indexing using color correlograms. Proceedings of CVPR. (1997) 762-768

12. H.Zhang etal,.: Image retrieval based on color features: an evaluation study. Proceedings of SPIE. Vol. 2606 (1995) 212-220

13. K.C.Ravishankar, B.G.Prasad, S.K.Gupta and K.K.Biswas.: Dominant Color Region Based Indexing Technique for CBIR. In proceedings of the International Conference on Image Analysis and Processing (ICIAP'99). Venice. Italy. Sept. (1999) $887-892$

14. X.Wan and C.J.Kuo.: A multiresolution color clustering approach to image indexing and retrieval. Proceedings of ICASSP. (1998)

15. R.C.Gonzalez and P.Wintz.: Digital Image Processing. 2nd Edition. AddisonWesley. Reading. Mass. (1987)

16. D.Mohamad, G.Sulong and S.S.Ipson.: Trademark Matching using Invariant Moments. Second Asian Conference on Computer Vision. 5-8 Dec, Singapore. (1995)

17. G.Cortelazzo etal.,: Trademark Shapes Description by String-Matching Techniques. Pattern Recognition. 27(8) (1994) 1005-1018

18. A.K.Jain and A.Vailaya.: Image Retrieval using Color and Shape. Second Asian Conference on Computer Vision. 5-8 Dec. Singapore. (1995) 529-533

19. R.Mehrotra and J.E.Gary.: Similar-Shape Retrieval in Shape Data Management. IEEE Computer. 28(9) (1995) 57-62

20. H.V.Jagadish.: A Retrieval Technique for Similar Shapes. Proceedings of ACM SIGMOD. Colorado. ACM. New York. May (1991) 208-217

21. G.Lu and A.Sajjanhar.: Region-Based Shape Representation and Similarity Measure Suitable for Content-Based Image Retrieval. Multimedia Systems. 7 (1999) $165-174$ 